\title{
INFLUÊNCIA DO PRÉ NATAL NA ESCOLHA DO TIPO DE PARTO: REVISÃO DE LITERATURA
}

\author{
INFLUENCE OF PRENATAL ON CHOICE OF TYPE OF BIRTHCHILD: LITERATURE REVIEW
}

\author{
Silas Santos Carvalho ${ }^{\mathrm{a}^{*}}$ \\ Orcid: http://orcid.org/0000-0001-8845-8902
}

\author{
Raiane Farias Nunes Cerqueira ${ }^{\text {t* }}$ \\ Orcid: http://orcid.org/0000-0003-1177-7520
}

ssc.academico@hotmail.comª, rfncerqueira@gmail.com ${ }^{\mathrm{b}}$

Universidade Estadual de Feira de Santana*,Faculdade Ateneu de Feira de Santana**
Data de submissão: 21/09/2019 Data de Aceite: 18/12/2019

\section{RESUMO}

Introdução: estudos recentes realizados no Brasil revelam elevadas taxas de partos cesáreos desnecessários e a influência da assistência pré-natal na decisão das mulheres acerca da escolha do tipo de parto. Objetivo: analisar na literatura nacional a influência do pré-natal na escolha do tipo de parto das gestantes brasileiras. Materiais e Métodos: estudo bibliográfico, de abordagem qualitativa, do tipo Revisão Integrativa da Literatura (RIL) realizado nas bases de dados Scientific Eletronic Library Online (SCIELO) e Literatura da América Latina e Caribe (LILACS) no período de 2009 a 2018, sendo selecionados e analisados 08 estudos. Os estudos apresentam fatores e visões que influenciam as gestantes a escolherem o tipo de parto. Resultados: Identificaram-se 8 artigos no período estudado. Os estudos apontam que a assistência pré-natal tem potencial influência na escolha da via de parto das gestantes, porém, a pouca orientação acerca das desvantagens de cada tipo de parto e as reais indicações da cesariana, podem transmitir a ideia infundada de que parto cesáreo é mais seguro. Conclusão: É fundamental o apoio dos profissionais de saúde durante o pré-natal, auxiliando a escolha por meio da educação em saúde sobre cada tipo de parto.

Palavras-chave: Humanização da assistência; cuidado pré-natal. parto; enfermagem obstétrica.

\begin{abstract}
Introduction: recent studies conducted in Brazil reveal high rates of not necessary cesarean deliveries and the influence of prenatal care on women's decision to choose the type of birthchild. Objective: analyze in the national literature the influence of prenatal care in choosing the type of birthchild of Brazilian pregnant women. Materials and methods: This is a bibliographical study, based on an Integrative Literature Review (RIL) available in the Scientific Electronic Library Online (SCIELO) and Latin American and Caribbean Literature (LILACS) databases in 2009 to 2017 being selected and analyzed 08 studies. The studies present factors and visions that influence the pregnant women to choose the type of birthchild. Results: We identified 8 articles in the period studied. Studies point out that prenatal care has a potential influence on the choice type of birthchild of pregnant women, but a low orientation on the advantages of each type of birthchild and as real indications of caesarean, can convey an unfounded idea of cesarean is safer. Conclusion: It is essential to support health professionals during prenatal care, helping the choice through health education on each type of delivery.
\end{abstract}

Keywords: Humanization of assistance; prenatal care. parturition; obstetric nursing 


\section{Introdução}

As consultas de pré-natal constituem um dos componentes essenciais para acompanhamento e monitorização da gestação, pois é possível detectar agravos de saúde ligados à atenção da saúde materno-fetal, consolidando os cuidados e condutas desde a concepção ao trabalho de parto e pós-parto, cuja finalidade é prevenir eventos adversos, identificar e tratar complicações ${ }^{1-5}$.

Mesmo sendo oferecidas gratuitamente pelo Ministério da Saúde (MS) as seis consultas de acompanhamento mínimo no pré-natal por gravidez, nem todas as mulheres têm frequentado as consultas marcadas, seja pelo desconhecimento da importância desse tipo de atendimento ou pela falta de comprometimento ou ainda, em virtude de algumas culturas não permitirem o acesso ${ }^{6-8}$.

É ideal também que no pré-natal o profissional preste as devidas orientações acerca do momento do trabalho de parto e parto, sendo essas informações fundamentais, pois previnem comportamentos que aumentem a ansiedade, os medos e as inseguranças no momento de parir, além de estimular a própria gestante a exercer a autonomia na escolha deste processo não deixando se influenciar a respeito de decisões que envolvem seu próprio corpo. São essenciais encontros para que se sanem as possíveis dúvidas, e propiciar que os conflitos vividos não interfiram de forma negativa nesse momento tão especial de nascimento de um filho ${ }^{9-12}$.

Existem duas vias de parto possíveis a qual sua escolha dependerá da avaliação obstétrica, são elas: parto cesáreo, somente realizado pelo médico obstetra em conjunto com sua equipe, por meio de uma conduta cirúrgica; e o parto normal simples em vértice que pode ser realizado tanto pelo médico quanto por um enfermeiro obstetra ${ }^{13,14}$.

Quando a gestante e o feto estiverem em risco e necessitarem de intervenções para sobreviver se faz o parto cesáreo que tem como finalidade intervir quando riscos são maiores diante dos benefícios do parto normal. No entanto, esse tipo de procedimento traz uma série de complicações tanto para a grávida quanto para o concepto, uma vez que a indicação inadequada pode favorecer a morbimortalidade materna e infantil ${ }^{15}$.
O parto normal é o mais indicado e seguro visto que o corpo feminino mantém uma preparação própria para a realização do mesmo, no entanto pode levar um período maior de trabalho de parto além de ser mais propenso à dor, o que acarreta na escolha pelo parto cesáreo na maioria das mulheres ${ }^{16}$.

Estudos recentes, acerca da assistência prénatal, realizados no Brasil demonstram que embora o Ministério da Saúde (MS) preconize o parto normal, os números de cesarianas aumentaram consideravelmente nos últimos anos ${ }^{15,17}$.

A elevação da taxa de cesarianas chega a $40 \%$ no Brasil, o que de certa forma é desnecessária e que está acima do limite de 15\% esperado pela Organização Mundial de Saúde (OMS). Enquanto em 2009 o país alcançava uma proporção de 50\% de partos cesáreos, em 2010, a taxa subiu para $52 \%$. Na rede privada, o índice de partos cesáreos chega a $82 \%$ e, na rede pública, $37 \%{ }^{15,17,18}$.

O interesse pela temática provém da relevância do problema no Brasil, onde nota-se que há certa influência da assistência no pré-natal na decisão das mulheres na escolha do tipo de parto. Justificase então a importância desta pesquisa como forma de descrever que os modelos de atenção ao parto e nascimento atuam auxiliando no processo de escolha das gestantes, que muitas vezes deixam de lado os partos ditos espontâneos e submetem-se cada vez mais a intervenções cirúrgicas.

No entanto, nota-se que apesar de tais medidas serem preconizadas na teoria, na prática observase que condutas intervencionistas têm sido cada vez mais empregadas sem uma indicação médica específica, o que acarreta em uma maior prevalência da realização de partos cesáreos.

Por essa razão surge o seguinte problema: Existe influência da assistência pré-natal na escolha do tipo de parto das gestantes brasileiras? Diante disso, o objetivo deste estudo foi analisar a influência do pré-natal na escolha do tipo de parto das gestantes brasileiras, segundo a literatura nacional.

\section{Metodologia}

Trata-se de uma revisão integrativa da literatura, que consiste na síntese e conclusões 
gerais de vários estudos, realizada de maneira sistemática e ordenada que permitiu a inclusão de assuntos comprovados para uma revisão completa do fenômeno analisado, tendo como eixo condutor a influência da consulta do prénatal na escolha da gestante ao tipo de parto. Incluindo também pontos de vista teóricos e contextuais, além de incorporar várias definições de conceitos, evidências e análises de problemas metodológicos ${ }^{19}$.

A questão de pesquisa delimitada foi: Existe influência da assistência pré-natal na escolha do tipo de parto das gestantes brasileiras?

Para localização e seleção dos estudos foi realizada a busca nas bases de dados SCIELO (Scientific Eletronic Library Online) e LILACS
(Literatura da América Latina e Caribe). Os critérios de inclusão foram: artigos publicados em português, no período compreendido entre 2009 e 2018, disponíveis integralmente e gratuitos, que abordassem definições claras quanto à temática do estudo. Utilizou-se como critério de exclusão: estudos de revisão, resultados de monografias, teses e dissertações e aqueles com duplicidades entre bases de dados.

Os Descritores em Ciências da Saúde (DeCS) utilizados foram: "humanização da assistência", "cuidado pré-natal", "parto" e "enfermagem obstétrica", de forma isolada e combinada. A busca foi realizada utilizando-se os critérios de inclusão e a amostra final foi constituída de 08 artigos, conforme Figura 1.

Figura 1 - Fluxograma do processo de busca dos artigos.

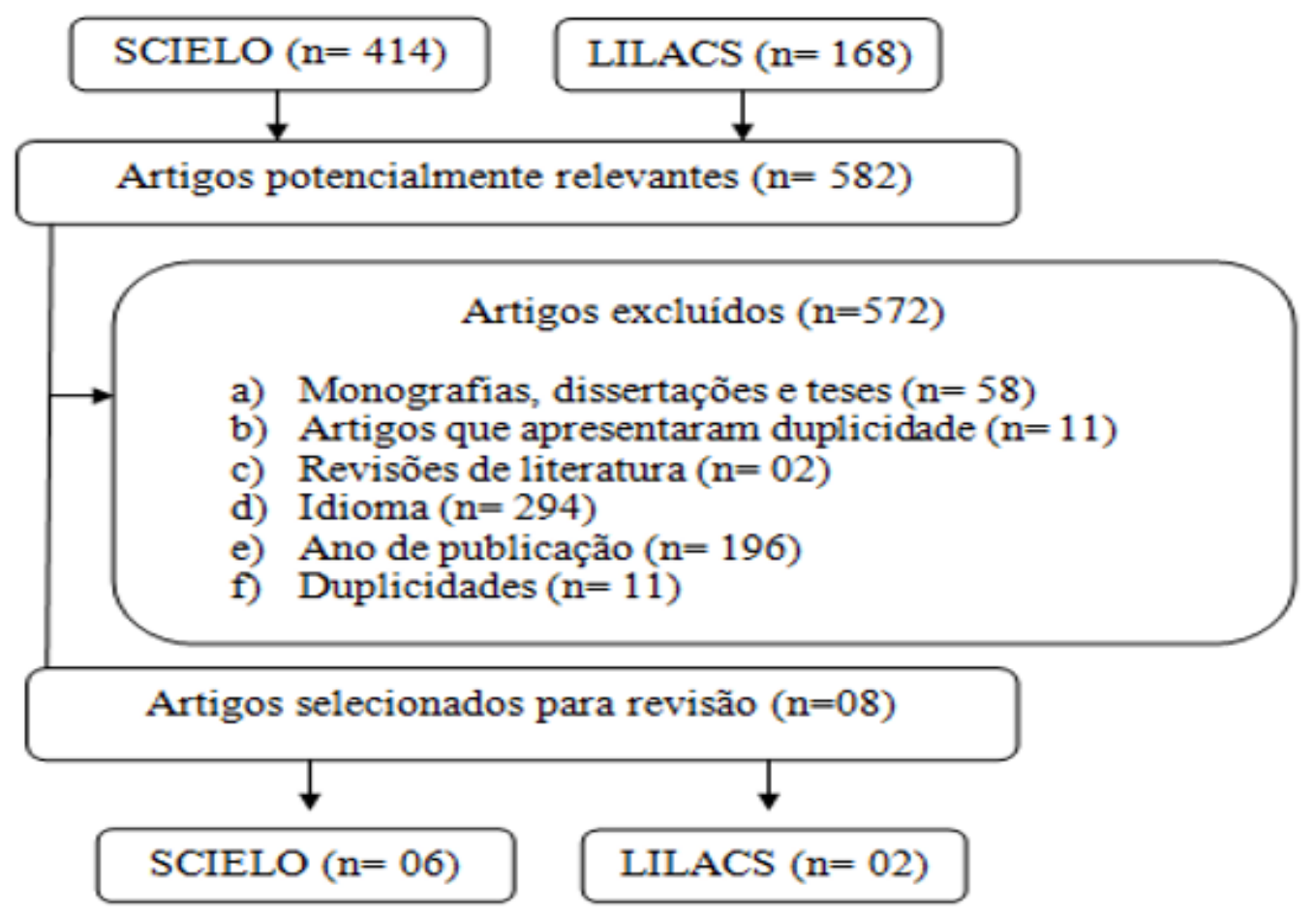

Fonte: Dados da pesquisa.

Após o levantamento dos artigos disponíveis procedeu-se a análise dos artigos, que consiste na interpretação e aproveitamento dos mesmos na produção científica, buscando direcioná-los ao proposto pelo objetivo da pesquisa ${ }^{20}$.
Essa revisão foi realizada apresentando como referência busca em artigos científicos, respeitando os critérios de elaboração que se baseia na Lei ${ }^{\circ}$ 9.610, de 19 de fevereiro de $1998^{21}$ que rege sobre os direitos morais e patrimoniais e os princípios éticos de combate ao plágio. 


\section{Resultados}

Foram identificados nas buscas 582 artigos. Retiraram-se 11 publicações por estarem duplicadas nas bases de dados e 561 estudos por não atenderem os critérios de inclusão. Após a leitura na íntegra dos 10 estudos, compôs-se a amostra final por 08 artigos. Extraíram-se destes, $75 \%$ (seis) da base de dados SCIELO e 25\% (dois) da LILACS.

Em relação ao tipo de revista nas quais foram publicados os estudos incluídos na revisão, $87,5 \%$ foram publicados em revista de enfermagem ou com foco em atenção materno infantil e 12,5\% estudos em revista de psicologia. Quanto ao tipo de delineamento de pesquisa dos estudos avaliados, evidenciaram-se na amostra 50\% do tipo descritivo qualitativo, $37,5 \%$ transversais quantitativos e $12,5 \%$ do tipo coorte. Em relação ao período de publicação, em 2018 apresentou 25\%; e nos anos 2009, 2010, 2013, 2014, 2015 e 2017 houve $12,5 \%$.

$\mathrm{O}$ quadro abaixo segue informativo com título, autores, ano de publicação e principais achados, para melhor visibilidade dos dados obtidos visando à reflexão crítica sobre a temática investigada.

Quadro 1 - Apresentação da síntese de estudos incluídos na revisão integrativa.

\begin{tabular}{|c|c|c|c|}
\hline Autor/Ano & Revista & Título & Principais achados \\
\hline $\begin{array}{l}\text { Mandarino NR et al, } \\
2009\end{array}$ & Cad. Saúde Pública & $\begin{array}{l}\text { Aspectos relacionados à } \\
\text { escolha do tipo de parto: um } \\
\text { estudo comparativo entre } \\
\text { uma maternidade pública e } \\
\text { outra privada, em São Luís, } \\
\text { Maranhão, Brasil }\end{array}$ & $\begin{array}{l}\text { A satisfação das parturientes foi elevada } \\
\text { com as duas vias de parto, porém o desejo } \\
\text { de repetir foi mais frequentemente referido } \\
\text { por aquelas submetidas ao parto vaginal } \\
\text { ( } 71,6 \% \text { vs. } 41,3 \% \text { na maternidade pública } \\
\text { e } 100 \% \text { vs. } 65,5 \% \text { na privada). }\end{array}$ \\
\hline Pires D et al, 2010 & $\begin{array}{l}\text { Rev. Brasil Saúde } \\
\text { Materno Infantil }\end{array}$ & $\begin{array}{l}\text { A influência da assistência } \\
\text { profissional em saúde na } \\
\text { escolha do tipo de parto: um } \\
\text { olhar socioantropológico na } \\
\text { saúde suplementar brasileira }\end{array}$ & $\begin{array}{l}\text { Entre os fatores que contribuíram para a } \\
\text { alta incidência de parto cesáreo ( } 81,8 \% \\
\text { das entrevistadas) estão: fatores culturais, } \\
\text { experiências anteriores, relações familiares } \\
\text { e a assistência pré-natal baseada na } \\
\text { biomedicina, a medicalização do processo } \\
\text { do parto, o medo da dor, a conveniência da } \\
\text { data marcada e um processo de negociação/ } \\
\text { orientação que se estabelece entre a } \\
\text { gestante e seus familiares e o profissional } \\
\text { médico. }\end{array}$ \\
\hline $\begin{array}{l}\text { Pinheiro BC, Bittar } \\
\text { CML, 2013 }\end{array}$ & Rev. Psicologia & $\begin{array}{l}\text { Expectativas, percepções e } \\
\text { experiências sobre o parto } \\
\text { normal: relato de um grupo } \\
\text { de mulheres }\end{array}$ & $\begin{array}{l}\text { A escolha do tipo de parto pelas puérperas } \\
\text { entrevistadas sofre influência de diferentes } \\
\text { mecanismos institucionais, individuais } \\
\text { e coletivos, de experiências prévias, do } \\
\text { poder médico e da família, da ausência } \\
\text { de ação dialógica no período pré-natal, do } \\
\text { interesse, curiosidade e bagagem cultural, } \\
\text { entre outras. }\end{array}$ \\
\hline $\begin{array}{l}\text { Domingues RM et al, } \\
2014\end{array}$ & Cad. Saúde Pública & $\begin{array}{l}\text { Processo de decisão pelo } \\
\text { tipo de parto no Brasil: } \\
\text { da preferência inicial das } \\
\text { mulheres à via de parto final }\end{array}$ & $\begin{array}{l}\text { Após o aconselhamento recebido durante a } \\
\text { assistência pré-natal, } 63,2 \% \text { das mulheres } \\
\text { acreditavam que, para uma gestação sem } \\
\text { complicações, o parto vaginal era o mais } \\
\text { seguro, } 6,3 \% \text { que o parto cesáreo era o mais } \\
\text { seguro, } 21,5 \% \text { que ambos eram seguros e } \\
9 \% \text { não se sentiam esclarecidas. }\end{array}$ \\
\hline
\end{tabular}




\begin{tabular}{|c|c|c|c|}
\hline Autor/Ano & Revista & Título & Principais achados \\
\hline $\begin{array}{l}\text { Nascimento RRP et al, } \\
2015\end{array}$ & $\begin{array}{l}\text { Rev. Gaucha de } \\
\text { Enfermagem }\end{array}$ & $\begin{array}{lcr}\text { Escolha } & \text { do tipo de } & \text { parto: } \\
\text { fatores } & \text { relatados } & \text { por } \\
\text { puérperas } & & \\
\end{array}$ & $\begin{array}{l}\text { A maioria das mulheres manifestou } \\
\text { preferência pelo parto normal devido à } \\
\text { recuperação rápida, menor dor esofrimento. } \\
\text { Porém a existência de interferências } \\
\text { familiares, as experiências prévias com } \\
\text { o parto e a interação profissional cliente } \\
\text { nas consultas pré-natais influenciaram na } \\
\text { escolha do tipo de parto. }\end{array}$ \\
\hline Silva ACL et al, 2017 & $\begin{array}{l}\text { Rev. Eletrônica de } \\
\text { Enfermagem }\end{array}$ & $\begin{array}{l}\text { Preferência pelo tipo de } \\
\text { parto, fatores associados à } \\
\text { expectativa e satisfação com } \\
\text { o parto }\end{array}$ & $\begin{array}{l}\text { O parto cesáreo foi a via de maior } \\
\text { prevalência, embora o parto normal tenha } \\
\text { sido o de maior preferência pela maioria } \\
\text { das gestantes. Primigestas e mulheres que } \\
\text { tiveram parto normal prévio apresentaram } \\
\text { maior desejo pelo desfecho normal. } \\
\text { A experiência anterior influenciou na } \\
\text { preferência pelo tipo de parto. }\end{array}$ \\
\hline $\begin{array}{l}\text { Kottwitz F, Gouveia HG, } \\
\text { Gonçalves AC, } 2018\end{array}$ & Escola Anna Nery & $\begin{array}{l}\text { Via de parto preferida por } \\
\text { puérperas e suas motivações }\end{array}$ & $\begin{array}{l}\text { A maioria }(77,6 \%) \text { das mulheres considerou } \\
\text { que o parto ao qual foram submetidas, seja } \\
\text { vaginal ou cesariana, não tinha riscos à sua } \\
\text { saúde e a do bebê. Porém, relataram que } \\
\text { há melhor recuperação pós-parto no parto } \\
\text { vaginal. Houve associação estatística entre } \\
\text { paridade e tipo de parto anterior com a via } \\
\text { de parto preferida. }\end{array}$ \\
\hline Martins APC et al, 2018 & $\begin{array}{l}\text { Rev. Bahiana de } \\
\text { enfermagem }\end{array}$ & $\begin{array}{l}\text { Aspectos que influenciam a } \\
\text { tomada de decisão da mulher } \\
\text { sobre o tipo de parto. }\end{array}$ & $\begin{array}{l}\text { As experiências vivenciadas pelos } \\
\text { familiares, por pessoas próximas e pela } \\
\text { própria gestante, bem como sentimentos e } \\
\text { sensações experimentadas, e a assistência } \\
\text { pré-natal recebida no decorrer do processo } \\
\text { foram alguns dos aspectos encontrados } \\
\text { e discutidos no presente estudo como } \\
\text { influenciadores na decisão da mulher. }\end{array}$ \\
\hline
\end{tabular}

Observa-se nos estudos selecionados que a assistência pré-natal tem potencial influência na escolha da via de parto das gestantes. Todavia, a pouca ou nenhuma orientação acerca das reais indicações de cesariana e as desvantagens de cada tipo de parto durante as consultas podem transmitir a ideia infundada de que o parto cesáreo é mais seguro e elevar a quantidade de cesáreas.

\section{Discussão}

O parto é visto como um processo psicossomático, no qual as escolhas das grávidas estão relacionadas não só com a própria evolução da gestação, mas também com o nível de informação da mulher, experiências prévias em outras gestações, e principalmente, a influência do profissional de saúde a respeito da decisão na hora de parir $^{17}$.
Historicamente um fator bastante relevante para a escolha da mulher é o aspecto psicológico na vivência da dor. Esta é vista como algo intolerável e extremamente doloroso fisicamente, o "preço a ser pago" pela maternidade, esperando assim antecipadamente que seu parto seja uma experiência permeada pela dor $^{22,23}$. Entretanto, estudo evidencia que a dor está relacionada ao perfil emocional do indivíduo, à interpretação que o mesmo dá a ela, bem como à expressão do sujeito sobre suas experiências sensoriais, afetiva e comportamental ${ }^{24}$.

Estudos evidenciam que mulheres que não têm as devidas orientações do pré-natal, no momento do parto agem instintivamente e sabem como agir, no entanto, há mulheres que necessitam de instruções norteadoras para se basear e assim conseguir manter a tranquilidade necessária para as contrações no momento de parir ${ }^{25,26}$. 
Acredita-se que a gestante necessita de conhecimentos prévios sobre a gravidez, a alimentação, o trabalho de parto (a respeito das contrações, como se comportar no momento do parto propriamente dito; posições em que deve ficar para participar e visualizar o parto). É a partir das possíveis respostas a essas e dentre outras indagações que a gestante vai assumir uma postura frente às decisões que deve tomar em relação a como conduzir da melhor forma seu parto. A maneira com que essas informações são recebidas - influenciadas pelo seu contexto social e econômico, sua personalidade e informações provindas do atendimento no pré-natal - norteia a tomada de decisão em relação à forma de usar seu corpo durante o parto ${ }^{16,27}$.

$\mathrm{O}$ enfermeiro apresenta uma assistência mais acolhedora transmitindo tranquilidade, segurança e conforto à gestante, nesse momento em que ela precisa de mais zelo por ser delicado, especial e único para a maior parte delas. Campanhas que estimulam o parto normal, incentivando a confiança, o controle da dor, que tragam informações verídicas e reais quanto aos prós e contras e segurança do procedimento, podem acarretar em uma maior adesão das mulheres ao parto vaginal e reduzir as solicitações de cesáreas e as cobranças jurídicas para os obstetras ${ }^{28-30}$.

Uma característica comum nas experiências citadas num estudo realizado com 81 gestantes que recebiam assistência de pré-natal ocorrida em quatro UBS no Município do Vale de Taquari, a respeito do parto normal e cesárea, foi a falta de informações sobre esses eventos para vivenciar o parto com segurança e autodeterminação ${ }^{31}$.

No século XIX o parto normal era tido como um processo fisiológico e natural com plenas possibilidades de acontecer na própria residência da gestante, sendo a grávida responsável pelo processo, com autonomia e participação em todo o transcorrer do parto, sendo assim respeitado o seu direito de mulher. O médico só era chamado em situações extremas quando a parteira não conseguia resolver o problema ${ }^{23,32,33}$.

Todavia, com o advento das pesquisas científicas em relação ao parto na idade média, verificou-se a necessidade da ciência exata no ramo da saúde da mulher, e constatou-se então que a mulher por ser frágil e precisar de acolhimento no momento da parturição precisava de medidas terapêuticas do profissional médico, deixando de lado o empirismo das parteiras e apregoando o modelo da medicalização do parto, mudando também o local de realização dos mesmos, deixando de ser o domicílio e passando a ser realizado nos hospitais $^{23,33}$.

Apesar de aumentar o risco de morbimortalidade para o binômio mãe/recémnascido os profissionais relatam que uma das maiores causas para a realização do parto cesáreo é a própria vontade das gestantes para que assim não sinta dor durante o processo, medo de não conseguir ter o parto, medo da forma como será conduzido pelos profissionais, ou então, não acarrete em modificações anatômicas da sua genitália e muita das vezes essa cesárea a pedido, ou seja, sem indicação clínica específica, não vem atrelada a orientações a respeito dos riscos do procedimento desde o parto ao nascimento ${ }^{13}$.

Conforme estudos, o número de partos cesáreos é elevado devido à rapidez do procedimento cirúrgico e por ocasionar menos dor. Por outro lado, o parto normal é considerado como um procedimento em que as mulheres relatam ser doloroso devido às técnicas empregadas, como a amniotomia e uso de ocitocina ${ }^{22,34}$.

Os fatores que influenciam na incidência de cesarianas são a remuneração do parto, aspectos culturais, falta de tempo dos obstetras e a forma como está estruturada a assistência hospitalar de saúde pública e a conveniada. Para o médico, o procedimento cirúrgico é melhor, pois no parto natural terá que dispor de muito tempo para atender de forma integral a gestante, deixando de lado suas outras atividades ${ }^{22,24,35}$.

Faz-se necessário citar que a dor aguda sem nada para amenizá-la ou justificá-la previamente faz com que muitas mulheres passem por experiências traumáticas, afirmando a crença de que o parto normal é munido exclusivamente de dores insuportáveis e que por isso o parto cesáreo seria a melhor opção ${ }^{36}$.

Nesse contexto, ressalta-se que três estudos $^{13,24,26}$ mencionam os manejos não farmacológicos ou boas práticas obstétricas baseadas em evidências científicas. Por meio dessas, os profissionais de saúde oferecem à mulher 
o apoio psicológico e emocional, as técnicas de relaxamento como massagens, música e outras alternativas que promovem alívio e conforto durante o trabalho de parto, tornando-o uma experiência menos dolorosa e mais gratificante. Essas medidas configuram ações que devem ser desenvolvidas durante a assistência pré-natal e podem fortalecer as mulheres na decisão pelo parto vaginal, aumentando sua confiança em sua capacidade de parir e de lidar com o processo da parturição ${ }^{13,26,27}$.

Investigações evidenciaram que as gestantes almejam um parto sem intercorrências, porém para a escolha da via de parto há a influência do medo da dor e das experiências relatadas por outras mulheres ${ }^{22,24,37,40}$.

O parto normal vai além da experiência física. Ele proporciona às mulheres força para lidar com a parturição, visando a dor como um aspecto intrínseco do ato de parir, transformando esse momento de sensação física e dolorosa em sentimento de amor, vivenciando o protagonismo feminino proporcionado pela maternidade ${ }^{27,38}$.

Em um estudo realizado com 190 puérperas sobre a preferência pelo tipo de parto em um Hospital do Triangulo Mineiro, observouse que $68,9 \%$ desejavam parto normal; destas, $55,7 \%$ positivaram a recuperação materna mais rápida, $14,5 \%$ acreditam que é mais saudável para mãe e recém-nascido; $8,4 \%$ citaram a dor como momentânea e 8,4\% gostariam de ter outros partos normais pela experiência positiva ${ }^{13}$.

Em outra investigação, as puérperas alegaram que foram orientadas muito superficialmente, em que não foram oferecidas informações sobre procedimentos adotados em cada tipo de parto, nem os aspectos psicológicos relacionados à experiência do parto ${ }^{24}$.

Apesar da maioria das gestantes desejarem parto normal, a maioria dos nascimentos ocorre por via alta, pois o modelo médico intervencionista traz como efeito cada vez menos partos naturais ${ }^{39,40}$.

No entanto, é de extrema relevância a ação educativa de qualidade durante o pré-natal, pois influencia na cadeia de opiniões e crenças acerca das vias de parto e, desse modo, promove a melhoria da autonomia da mulher na escolha final $^{26,36,40}$.

\section{Conclusão}

A presente revisão integrativa reafirma a importância que a consulta de pré-natal tem na vivência do parto de uma mulher. A vulnerabilidade da mulher desencadeada pelo processo de parturição, somada à falta de conhecimento, faz com que a gestante não valorize sua autonomia, seu potencial de decisão frente às evidências médicas.

Conforme os estudos analisados, verificou-se que existem fatores que interferem na escolha do tipo de parto pelas gestantes, entre eles: as experiências prévias, as opiniões de amigos/ familiares e principalmente a ausência do diálogo acerca do assunto no período pré-natal. Diante disso, faz-se necessário que nas consultas de prénatal, esses fatores sejam discutidos e as gestantes recebam informações consistentes e seguras a fim de terem autonomia para tomada de decisão sobre a escolha do tipo de parto.

Pretendeu-se, assim, com este estudo, fornecer subsídios para políticas de expansão da cobertura do acompanhamento pré-natal e implementação de medidas que propiciem a melhoria da qualidade do atendimento, especialmente no que se refere às orientações prestadas à gestante, as quais vão norteá-la na escolha da via de parto.

Diante disso, é imprescindível a participação do enfermeiro na atenção ao ciclo gravídico puerperal, pois esse profissional desempenha um papel fundamental no processo educativo, além de assistir à mulher com qualidade e de forma mais humanizada. Para isso, é preciso que os profissionais de saúde, que atendem diretamente à gestante, desenvolvam a escuta, a disponibilidade para questionamentos que favoreçam uma relação de confiança, a fim de que o vínculo e confiança com essa mulher possam ocorrer.

\section{Referências}

1. Viellas EF, Domingues RMSM, Dias MAB, Gama SGN, Filha MMT, Costa JV, Bastos MHMC. Assistência prénatal no Brasil. Cad. Saúde Pública. 2014; 30(1):85-100. 
2. Brasil. Ministério da Saúde. Gestação de alto risco: manual técnico. Secretaria de Atenção à Saúde. Departamento de Ações Programáticas Estratégicas. 5. ${ }^{a}$ ed. Brasília: Ministério da Saúde. 2010. Disponível em: URL: http:// bvsms.saude.gov.br/bvs/publicacoes/gestacao_alto_risco. pdf

3. Brasil. Ministério da Saúde. Atenção ao pré-natal de Baixo Risco. Manual técnico/ Ministério da Saúde. Secretaria de Atenção à Saúde. Departamento de Ações Programáticas Estratégicas. 1. ${ }^{\text {a }}$ Ed. Brasília: Ministério da Saúde. 2013. Disponível em: URL: https://bvsm.saúde.gov.br/bvs/ publicacoes/manual_atencao_prenatal_baixo_risco.pdf.

4. Melo DBL, Rocha DFJS, Moreira SFLR, Rodrigues OIV, Silva SJ, Feitosa ANA. Fatores que predispõem para a gestação de alto risco. Revista Interdisciplinar em Saúde. 2017; 4(1): 72-86.

5. Nunes TJ, Gomes ORK, Rodrigues PTM, Mascarenhas MDM. Qualidade da assistência pré-natal no Brasil: Revisão de artigos publicados em 2005 a 2015. Cad. Saúde Coletiva. 2016;24(2):252-261.

6. Brasil. Ministério da Saúde. Humanização do parto e do nascimento. Brasília: Ministério da Saúde. 2014. 465p. Disponível em: URL: http://www.redehumanizasus. net/sites/default/files/caderno_humanizasus_v4_ humanizacao_parto.pdf-12-03-2016.

7. Lima LR. Relação do perfil do acompanhamento nas consultas de pré-natal com os desfechos maternos e perinatais. 2017. 97 f. Dissertação (Mestrado em Saúde Pública) - Faculdade de Medicina, Universidade Federal do Ceará, Fortaleza, 2017.

8. Rocha CA, Andrade SG. Atenção da equipe de enfermagem durante o pré-natal: Percepção das gestantes atendidas na rede básica de Itapuranga - GO em diferentes contextos sociais. Revista Enfermagem contemporânea. Goiás- GO, 2017.

9. Rodrigues IR, Rodrigues DP, Ferreira MA, Pereira MLD, Barbosa EMG. Elementos constituintes da consulta de enfermagem no pré-natal na ótica de gestantes. Revista Rene. 2016;17(6): 774-81.

10. Duarte SJH, Almeida EP. O papel do enfermeiro do programa saúde da família no atendimento pré-natal. Rev. Enferm. Cent-Oeste Min. 2014;4(1):1029-35.

11. Rios CTF, Vieira NFC. Ações educativas no pré-natal: reflexão sobre a consulta de enfermagem como um espaço para educação em saúde. Ciênc. saúde coletiva. 2007;12(2): 477-486

12. Tostes NA, Seidl EMF. Expectativas de gestantes sobre o parto e suas percepções acerca da preparação para o parto. Temas psicol. 2016;24(2):681-693.

13. Silva ACL, Felix HCR, Ferreira MBG, Wysocki AD, Contim D, Ruiz MT. Preferência pelo tipo de parto, fatores associados à expectativa e satisfação com o parto. Rev. Eletr. Enf. 2017; 19:1-11.
14. Zanatta E, Pereira CRR, Alves APA. Experiência da maternidade pela primeira vez: as mudanças vivenciadas no tornar-se mãe. Pesqui. prát. Psicossociais. 2018; 13(1):1-16.

15. Leal MC, Gama SGN. Nascer no Brasil. Cad. Saúde Pública. 2014; 30(1). Disponível em: URL: <http://www. scielo.br/scielo.php?script $=$ sci_arttext\&pid $=$ S0102311X2014001300001\&lng=pt\&nrm=iso\&tlng=pt>.

16. FEBRASGO. Manual de gestação de alto risco. Comissões nacionais especializadas de ginecologia e obstetrícia, 2011.

17. Haddad SEMT, Cececatti JG. Estratégias dirigidas aos profissionais para a redução das cesáreas desnecessárias no Brasil. Rev. Bras. Ginecol. Obstet. 2011;33(5):252-262.

18. Giraldi A, Zorzetto R. Antes da hora. Cesarianas contribuem para o nascimento de bebês imaturos. Revista Pesquisa FAPESP. Ed. 228, p. 20, 2015.

19. Souza MT, Silva MD, Carvalho R. Revisão integrativa: o que é e como fazer. Einstein. 2010;8(1 Pt 1):102-06.

20. Soares CB, Hoga LAK, Peduzzi M, Sangaleti C, Yonekura T, Silva DRAD. Integrative review: concepts and methods used in nursing. Rev esc enferm USP. 2014; 48(2):335-45.

21. Brasil. Lei $\mathrm{n}^{\circ}$ 9.610, de 19 de fevereiro de 1998. Altera, atualiza e consolida a legislação sobre direitos autorais e dá outras providências. Diário Oficial [da República Federativa do Brasil], Brasília [online], 20 fev. 1998.

22. Pires D, Fertonani HP, Conill EM, Matos TA, Cordova FP, Mazur CS. A influência da assistência profissional em saúde na escolha do tipo de parto: um olhar socioantropológico na saúde suplementar brasileira. Rev. Bras. Saude Mater. Infant. 2010; 10(2):191-197.

23. Velho MB, Santos EKA, Collaço VS. Parto normal e cesárea: representações sociais de mulheres que os vivenciaram. Rev Bras Enferm. 2014;67(2): 282-9.

24. Pinheiro BC, Bittar CML. Expectativas, percepções e experiências sobre o parto normal: relato de um grupo de mulheres. Fractal, Rev. Psicol. 2013; 25(3):585-602.

25. Mandarino NR, Chein CBM, Junior MCF, Brito OML, Lamy CZ, Nina SJV, Mochel GE, Neto FAJ. Aspectos relacionados à escolha do tipo de parto: um estudo comparativo entre uma maternidade pública e outra privada, em São Luís, Maranhão, Brasil. Cad. Saúde Pública. 2009;25(7):1587-1596.

26. Martins APC, Jesus NVM, Junior PPP, Passos MC. Aspectos que influenciam a tomada de decisão da mulher sobre o tipo de parto. Rev. baiana enferm. 2018;32.

27. Silva SPC, Prates RCG, Campelo BQA. Parto normal ou cesariana? Fatores que influenciam na escolha da gestante. Rev Enferm UFSM. 2014;4(1):1-9

28. Rezende JF, Montenegro CAB. Obstetrícia fundamental. 12a edição. Rio de Janeiro: Guanabara-Koogan, 2011. 
29. Oliveira CE, Barbosa MS, Melo PES. A importância do acompanhamento pré-natal realizados por enfermeiros. Revista Científica FacMais. 2016;7(3):25-38.

30. Carvalho SS, Oliveira BR, Bezerra ISA. Importância das orientações sobre trabalho de parto nas consultas de pré-natal: revisão de literatura. Rev. Educ. Saúde 2019;7(2):142-150.

31. Weidle WG, Medeiros CRG, Grave MTQ, Bosco SMD. Escolha da via de parto pela mulher: autonomia ou indução?. Cad. saúde colet. 2014;22(1):46-53.

32. Leister N, Riesco MLG. Assistência ao parto: história oral de mulheres que deram à luz nas décadas de 1940 a 1980. Texto contexto - enferm. 2013;22(1):166-174

33. Ministério da Saúde. Parto, aborto e puerpério: assistência humanizada à mulher. Brasília: Ministério da Saúde; 2001.

34. Nucci M, Nakano AR, Teixeira LA. Ocitocina sintética e a aceleração do parto: reflexões sobre a síntese e o início do uso da ocitocina em obstetrícia no Brasil. História, Ciências, Saúde. 2018;25(4):979-998.

35. Cardoso PO, Alberti LR, Petroianu A. Morbidade neonatal e maternas relacionada ao tipo de parto. Revista Ciência \& Saúde Coletiva. 2010;15(2).

36. Domingues RM, Soares M, Dias MAB, Pereira MN, Torres JA, Orsi E, Pereira APE, Schilithz AOC, Leal MC. Processo de decisão pelo tipo de parto no Brasil: da preferência inicial das mulheres à via de parto final. Cad. Saúde Pública. 2014;30(1):101-116.

37. Feitosa RMM, Pereira RD, Souza TJCP, Freitas RJM, Cabral SAR, Souza LFF. Factors that influence the choice of birth type regarding the perception of puerperal women. Rev Fund Care Online. 2017; 9(3):717-726.

38. Gama AS, Giffin KM, Angulo-Tuesta A, Barbosa GP, d'Orsi E. Representações e experiências das mulheres sobre a assistência ao parto vaginal e cesárea em maternidades pública e privada. Cad. Saúde Pública. 2009;25(11):2480-2488.

39. Kottwitz F, Gouveia HG, Goncalves AC. Via de parto preferida por puérperas e suas motivações. Esc. Anna Nery. 2018;22(1).

40. Nascimento RRP, Arantes LS, Souza CDE, Contrera L, Sales APA. Escolha do tipo de parto: fatores relatados por puérperas. Rev. Gaúcha Enferm. 2015;36:119-126.

\section{Como citar este artigo:}

Carvalho SS, Cerqueira RFN. Influência do pré natal na escolha do tipo de parto: revisão de literatura. Rev. Aten. Saúde. 2020; 18(63): 120-128. 